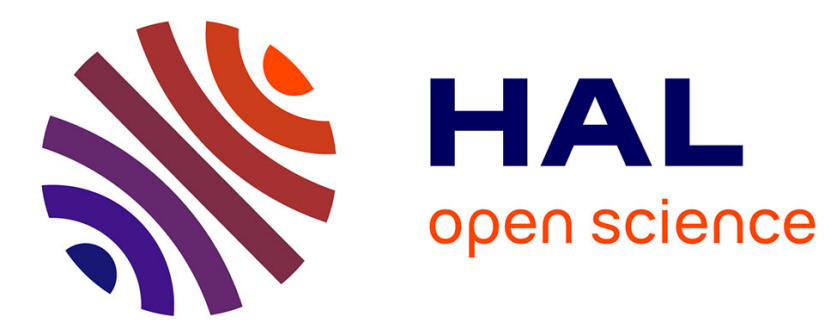

\title{
Figures littéraires d'exilés, réfugiés, migrants en classe de Quatrième
}

\author{
Virginie Brinker, Sandrine Meslet
}

\section{To cite this version:}

Virginie Brinker, Sandrine Meslet. Figures littéraires d'exilés, réfugiés, migrants en classe de Quatrième. Le Français Aujourd'hui, 2017, 197, pp.73-84. 10.3917/lfa.197.0073 hal-01609528

\section{HAL Id: hal-01609528 \\ https://u-bourgogne.hal.science/hal-01609528}

Submitted on 20 Oct 2021

HAL is a multi-disciplinary open access archive for the deposit and dissemination of scientific research documents, whether they are published or not. The documents may come from teaching and research institutions in France or abroad, or from public or private research centers.
L'archive ouverte pluridisciplinaire HAL, est destinée au dépôt et à la diffusion de documents scientifiques de niveau recherche, publiés ou non, émanant des établissements d'enseignement et de recherche français ou étrangers, des laboratoires publics ou privés. 


\title{
FIGURES LITTÉRAIRES D’EXILÉS, RÉFUGIÉS, MIGRANTS EN CLASSE DE QUATRIËME
}

Construire une « lecture responsable»

\author{
Virginie Brinker, Sandrine Meslet
}

Armand Colin | « Le français aujourd'hui »

$2017 / 2 \mathrm{~N}^{\circ} 197$ | pages 73 à 84

ISSN 0184-7732

ISBN 9782200931148

DOI 10.3917/lfa.197.0073

Article disponible en ligne à l'adresse :

https://www.cairn.info/revue-le-francais-aujourd-hui-2017-2-page-73.htm

Distribution électronique Cairn.info pour Armand Colin.

(C) Armand Colin. Tous droits réservés pour tous pays.

La reproduction ou représentation de cet article, notamment par photocopie, n'est autorisée que dans les limites des conditions générales d'utilisation du site ou, le cas échéant, des conditions générales de la licence souscrite par votre établissement. Toute autre reproduction ou représentation, en tout ou partie, sous quelque forme et de quelque manière que ce soit, est interdite sauf accord préalable et écrit de l'éditeur, en dehors des cas prévus par la législation en vigueur en France. Il est précisé que son stockage dans une base de données est également interdit. 


\section{FIGURES LITTÉRAIRES D'EXILÉS, RÉFUGIÉS, MIGRANTS EN CLASSE DE QUATRIÈME}

\section{CONSTRUIRE UNE « LECTURE RESPONSABLE »}

\section{Virginie BRINKER}

Université de Bourgogne Franche-Comté

Centre pluridisciplinaire textes et cultures - ÉA 4178

\section{Sandrine MESLET}

Collège Jules Ferry

Villeneuve Saint-Georges

\section{Une éthique de la responsabilité sans instrumenter les textes?}

Aux prises avec une actualité sensible (Legardez et Simmoneaux 2006), nous souhaitions, dans le cadre de l'objet d'étude issu des nouveaux programmes de Quatrième "Individus et société : confrontation de valeurs ", conduire les élèves à s'interroger sur la figure du migrant construite par une œuvre littéraire. Cette séquence faisait directement suite à la courte étude d'un groupement de textes autour de la notion de valeurs, confrontant les valeurs des personnages à celles de leur famille ou encore de la société. L'enjeu consistait cette fois-ci à tenter de contrecarrer certaines représentations médiatiques, approximatives, parfois truffées de préjugés, concernant un thème à la fois plus précis et plus épineux. Il nous semblait pour ce faire qu'une œuvre francophone devait être mobilisée, offrant un point de vue décentré vis-à-vis de la société française et permettant de prendre un certain recul vis-à-vis des discours sur ladite " crise des migrants ». Des chercheurs en littératures francophones, à l'instar de C. Mazauric (2012), ont en effet révélé comment un certain nombre de textes issus de ce corpus opèrent une véritable inversion de perspective. Ces œuvres dessinent notamment le portrait du migrant en aventurier (Kandé 2011), ou dépouillent sa figure des connotations négatives qui l'entachent soit en réévaluant la figure du passeur, soit en relativisant les attentes des personnages ${ }^{1}$.

\footnotetext{
1. Nous rejoignons ici le propos de C. Mazauric lorsqu’elle écrit : «Vouloir croire en outre que tous les migrants seraient portés par une vision idéale de l'Europe, terre de prospérité et de liberté, c'est aussi, de la part des Européens, se bercer d'une illusion narcissique consistant
} 
La lecture de l'appel à contributions pour le numéro 197 du Français aujourd'hui a su stimuler notre réflexion et nous servir de garde-fou, tout en aiguisant le choix de l'œuvre à faire lire aux élèves. Nos partis pris et notre démarche ont ainsi été déterminés par certains passages de l'argumentaire de ce numéro. Le premier rappelait que «faire réfléchir les élèves aux valeurs des sociétés apparait donc plus que jamais aujourd'hui comme une des missions de l'école, mais cet objectif peut poser problème dès qu'on l'associe aux productions artistiques et notamment à la littérature. Celle-ci ne risquet-elle pas d'être instrumentalisée pour servir les objectifs - si légitimes soient-ils - de l'enseignement moral et civique ? ». Dans un autre passage les coordonnatrices se demandaient « comment exercer une éthique de la responsabilité sans instrumenter les textes ». De telles questions ont guidé notre réflexion ${ }^{2}$.

Le choix de l'œuvre théâtrale de M. Bey, Tu vois c'que j'veux dire? (2013), s'est alors imposé. M. Bey, femme de lettres qui est née et vit en Algérie, a écrit de nombreux romans, certains traitant de l'Algérie des années 1990 faisant face à l'islamisation radicale ${ }^{3}$, d'autres ayant pour objet la mémoire contemporaine de la guerre d'Algérie ${ }^{4}$, mais aussi des poèmes (Sahara, mon amour 2005), des nouvelles (Nouvelles d'Algérie 1998) et bien sûr du théâtre.

$T u$ vois c'que je veux dire? est une courte pièce qui a été créée à SaintÉtienne dans le cadre d'un projet de rencontres théâtrales en milieu scolaire (Cinq petites pièces africaines pour une comédie) en 2005, avant d'être montée en 2009 par J. Carmichael, directrice de la Compagnie Théâtr'elles, qui préface le texte publié. Ce qui nous intéressait au plus haut point était que cette pièce mettait en jeu - comme le laisse déjà percevoir le titre - une véritable dramaturgie de l'implicite laissant d'emblée au lecteur la possibilité d'investir librement le texte de ses propres valeurs. En effet, elle met en scène deux personnages, Farid et Kamel, deux jeunes Algériens qui ont décidé d'émigrer vers les rives nord de la Méditerranée et attendent leur passeur, une nuit, sur le port. L'identité des personnages n'est toutefois pas immédiatement révélée et leur projet non plus, a fortiori. Tu vois c'que j'veux dire?, titre de l'œuvre, est en effet un leitmotiv de la pièce, traduisant justement ses non-dits. Tout l'implicite lié aux ressentis des personnages, aux raisons profondes de leur exil, semble en effet se situer dans une sorte de

à se mirer dans le regard des autres - un regard que l'on a construit à leur place » (2012 : 329).

2. La démarche proposée par la suite concernant le travail sur la lecture interprétative et la compréhension fine d'une œuvre littéraire s'inscrit dans les réflexions didactiques menées, notamment, dans les ouvrages suivants : M.-F. Bishop et A. Rouxel (2007) ; R. Goigoux et S. Cèbe (2009) ; P. Joole (2008) ; A. Rouxel et G. Langlade (2004).

3. Au commencement était la mer, Paris, Marsa éditions, 1996 ; Cette fille-là, La Tourd'Aigues, éditions de l'Aube, 2001 ; Entendez-vous dans les montagnes, La Tour-d'Aigues, éditions de l'Aube, 2002.

4. Bleu, blanc, vert, La Tour-d'Aigues, éditions de l'Aube, 2007 ; Puisque mon cour est mort, La Tour-d'Aigues, éditions de l'Aube, 2010. 
sous-texte que suggèrent les points de suspension dans les paroles de Farid, notamment, ou dans la façon dont les personnages s'interrompent.

\section{Permettre aux élèves-lecteurs d'investir le texte de leurs propres valeurs}

Nous avons donc pris le parti de ne pas donner la pièce à lire intégralement aux élèves, ni même de la leur laisser à disposition, mais de découvrir le texte collectivement par le biais d'une lecture suivie. Ce n'est qu'en fin de séquence, pour des exercices d'écriture globaux, que la série d'ouvrages leur a été distribuée. Le recours aux courts écrits intermédiaires et individuels des élèves (du type " «carnet de lecture ») a également été systématisé au fil de la séquence pour recueillir leurs représentations et interprétations au fil du texte. Le profil des élèves explique, en partie, cette organisation. En effet, l'établissement situé en Réseau d'éducation prioritaire (REP +) mêlait des lecteurs précaires et des élèves plus experts, issus de la classe bilangue de l'établissement qui commence en Sixième et disparait à la fin de la Cinquième. Cette hétérogénéité rendait difficile la lecture personnelle suivie : il fallait créer une appétence pour la suite de l'histoire dans le but d'aider les lecteurs précaires à dépasser leurs difficultés de lecture, voire de décodage (Tauveron 2004). En effet, si ces derniers ne parvenaient pas à un niveau satisfaisant de fluence, le sens du texte leur échapperait. Il fallait alléger leur entrée dans le texte d'un certain nombre de difficultés pour leur permettre de se concentrer sur les compétences de lecture et d'interprétation que nous souhaitions travailler avec eux.

Dans un premier temps, il s'agissait donc de laisser les élèves imaginer qui étaient les protagonistes, où, quand et dans quelle situation ils se trouvaient. Nous souhaitions à ce moment-là comprendre dans quelle mesure les élèves pouvaient se sentir proches des personnages sans connaitre encore leur statut de migrants. Ceci permettait de miser, ultérieurement, sur la révélation de leur condition, en faisant alors verbaliser aux élèves ce qui se jouait sur le terrain des valeurs. Il s'agissait surtout de leur permettre, dans un raisonnement métatextuel, de revenir sur leurs premières interprétations de lecture et d'appréhender le choix de la dramaturge de ne pas révéler la situation et l'identité des protagonistes dès le départ.

La première séance a donc été consacrée à l'investissement par chaque élève de son interprétation de la scène d'exposition. Nous avons fait le choix, qui peut être discutable, de tronquer cet extrait, de façon à reculer au maximum la découverte du statut de « migrants » des personnages. Il nous semble toutefois que nous n'avons pas dénaturé le texte puisque la phrase que nous n'avons finalement pas retenue ${ }^{5}$ restait implicite et que ce trait

5. "Même nous, s'ils pouvaient, ils nous rayeraient des listes... sans grande perte pour le pays» (Bey $2013: 20)$. 
d'écriture, souligné par l'emploi récurrent des déictiques et des points de suspension, apparaissait comme une caractéristique majeure de la pièce.

Nuit.

Une rue très faiblement éclairée bordée de grands bâtiments sombres.

Deux jeunes gens. Farid et Kamel. Ils marchent assez rapidement. Ils discutent. La rue est déserte.

Tout d'abord, on n'entend pas ce qu'ils se disent. Puis leurs paroles deviennent de plus en plus distinctes au fur et à mesure qu'ils avancent vers le public.

Farid. - Je sais... je sais... mais... dis-moi, tu crois qu'on a des chances de... Kamel (Il s'arrête. Il l'attrape par le bras.). - Attends, écoute-moi, Farid ! Je te l'ai déjà dit. Y a des mots qu'il vaut mieux ne pas prononcer! Encore moins aujourd'hui, ici et maintenant! Celui-là, par exemple, tu évites, s'il te plait, ça pourrait nous porter malheur! Chance! Et puis quoi encore? La chance, on l'appelle pas! On la nomme pas. On sait même pas que ça existe. La preuve, c'est qu'on est là ce soir !

Ils se remettent à marcher.

Farid. - J'ai quand même un peu... un peu peur...

Kamel. - Ça aussi tu effaces! Si ça continue, je vais te faire une liste. Peur, chance, espoir, demain, réussite... et tous les mots comme ça. On y a pas droit pour le moment. On verra plus tard, quand on sera arrivés... si on arrive... [...] Et même si on sait pas où on va, l'essentiel c'est d'y aller, non ? En attendant, dépêche-toi. Il va sûrement pas nous attendre!

Farid. - Mais... on a le temps... il nous a dit minuit !

Kamel. - Je sais, je sais, mais vaut mieux être en avance, vaut mieux pas risquer.

(Bey : 17-21)

À partir de la lecture silencieuse de cet extrait et de sa projection au tableau, il était ainsi demandé à chaque élève de répondre individuellement et à l'écrit, de façon succincte, aux questions suivantes :

Qui est Farid ? Qui est Kamel ?

Que font-ils?

Que pourrait-il se produire?

Les premières réponses collectées font état de quatre interprétations distinctes.

On peut évacuer pour la nécessité du propos ici (même s'il nous a fallu y revenir en pratique) les réponses de quatre élèves convaincus qu'il s'agissait de jeunes gens s'apprêtant à jouer au théâtre ("Ce sont des acteurs de thêâtre ", écrit Youcef M.), trompés par la formule " au fur et à mesure qu'ils avancent vers le public ${ }^{6}$ » de la didascalie initiale qu'ils n'ont pas reconnue. Il faut dire que seule la présence des italiques pouvait véritablement aider à identifier cette convention propre au genre théâtral. En l'absence notable de découpage du texte en scènes et en actes numérotés, s'opérait ainsi

6. Nous soulignons. 
dans l'esprit de ces élèves, moins familiarisés avec la lecture de pièces, une confusion entre personnages et comédiens.

Deux autres interprétations globales, à vrai dire les plus répandues, font état de représentations antagonistes. Prenons ainsi l'exemple de Shangari, pour laquelle ce sont deux frères qui rentrent chez eux après avoir eu la permission de minuit, mais sont perdus et ont peur de la réaction de leur père : cette interprétation est à peu près partagée par quatre autres élèves. Yanis imagine quant à lui que ce sont deux jeunes gens qui cherchent à acheter de la drogue, tandis que Tierno pense qu'ils vont commettre un braquage, suivi sur ce point par Indira imaginant qu'ils font partie d'un gang et craignent d'être tués. Même si les stéréotypes télévisuels, notamment ceux des séries policières, semblent ici jouer à plein, il est notable qu'une partie de la classe semble s'identifier aux jeunes gens, envisageant des situations plausibles pour des adolescents, et qu'une autre les identifie comme êtres de fiction, d'emblée disqualifiés et envisagés comme des "délinquants ». À l'échelle de la classe, notons que c'est plutôt l'identification qui l'emporte : un groupe de six autres élèves (Kilian, Cumba, Aminata, Ousmane, Lucie et Shimona), sans trancher sur la situation précise des personnages, imagine qu'il s'agit de deux amis menacés par quelque chose, sans toutefois statuer sur la nature de la menace. Ce qui l'emporte dans leurs écrits intermédiaires est le sentiment de peur, un sentiment qu'ils partagent avec les personnages.

La découverte de la suite du texte (Bey 2013 : 21-25), lors de la séance $\mathrm{n}^{\circ} 3^{7}$, met au jour la situation, mais pas immédiatement. En dépit de plusieurs indices tels que la "tempête ", les "grosses vagues ", la « mer ", le "vent ", l'absence de travail et d'avenir évoquée par les personnages, le pressentiment de la mère de Farid de ne plus le revoir, l'absence d'attache de Kamel qui dit être orphelin ou encore la mention de "valises ", les premiers écrits intermédiaires de la plupart des élèves passent à côté de la situation de ces jeunes migrants. Beaucoup s'arrêtent sur les liens des personnages avec leur famille, ou bien sur le fait qu'ils ont amassé difficilement de l'argent, mais tous, quasiment, restent dans la paraphrase du texte, son sens dénoté, et évacuent la question du départ. L'écrit d'Aminata est ici très représentatif : «Kamel n’a pas de bons rapports familiaux. Il y a des problèmes météorologiques. Ils ont accumulé difficilement de l'argent ". L'enjeu de notre consigne ("Qu'apprend-on de nouveau dans cet extrait ?») était de faire réaliser aux élèves le décalage entre le passage inaugural et la suite du texte, mais sa formulation ouverte n'a pas conduit tous les élèves à dépasser la reformulation des explicites. Aïcha, Marie et Chimène sont les seules à parler du fait que les personnages vont partir en mer. C'est au moment de la mise en commun que Salim, un élève généralement silencieux,

7. Nous présentons ici le contenu de la séance 3 , avant celui de la séance 2 , pour expliciter les enjeux de la révision de leur lecture par les élèves. Nous, enseignantes, souhaitions que les élèves prennent le temps de réaliser le processus nécessaire au renforcement de leurs stratégies de lecture, sans une rectification magistrale immédiate et malvenue. 
lance « ils fuient leur pays »; il va alors justifier sa réponse en reprenant un à un tous les indices mentionnés plus haut. Après cet échange collectif, dans une nouvelle synthèse demandant à chacun si sa position a évolué à l'issue de l'échange, deux élèves sur seize ne reprennent pas l'idée d'exil évoquée par Salim. Kilian reste focalisé sur les questions familiales, tandis que Tierno parle simplement de "fugue ». D'autres, en revanche, commencent à greffer sur ces premières hypothèses d'autres représentations ne figurant à aucun moment dans le texte, quant à l'explication de ce départ. Rachid écrit ainsi " peut-être à cause de la guerre dans leur pays " et Marie " car leur pays est trop pauvre ». Ces élèves réinvestissent peut-être ici les discours médiatiques vus ou entendus, sans pour autant parvenir à justifier ces interprétations. Nous, enseignantes, décidons alors qu'une séance ultérieure sera consacrée à l'étude du texte «Baignade interdite » de P. Claudel (2015), permettant à la fois de réfléchir sur les représentations que l'on se forge des migrants, et de voir comment un texte littéraire peut se jouer des postures idéologiques. Nous y reviendrons.

\section{Construire et questionner l'effet-personnage}

Entretemps, pour construire une démarche de "lecture responsable», outre l'orchestration d'une fausse piste et de sa révision, la mise en question de l'«effet-personnage " (Jouve 1992) nous a semblé un bon outil pour permettre, par la suite, aux élèves de prendre du recul par rapport à leur posture de lecteur empathique. Cela a fait l'objet de notre deuxième séance. Lors de la formulation des premières hypothèses sur l'identité et la situation des personnages, une seule élève, Valérie, avait proposé un écrit intermédiaire un peu différent envisageant une séparation imminente des deux protagonistes. Elle avait été en effet prioritairement sensible aux différences de caractère des deux personnages, Kamel apparaissant comme plus velléitaire, autoritaire et sûr de lui, face à un Farid très craintif. Nous avons alors décidé de saisir cette opportunité pour travailler sur la caractérisation des personnages par leurs discours.

De fait, en séance 2, nous demandons aux élèves de rajouter des didascalies (gestes, ton...) dans le but de mieux faire apparaitre l'identité et la situation des personnages, avec pour objectif de saisir l'opportunité offerte par le texte théâtral de pouvoir "jouer " les personnages, et ce afin de mieux cerner leur caractère et leurs motivations intimes. Plusieurs binômes se montrent volontaires pour mettre en espace la lecture à haute voix de l'extrait. De leur côté, les spectateurs sont chargés de mettre des mots et du sens sur l'interprétation qui vient d'être donnée de l'extrait, indépendamment du projet initial du binôme qui la propose. Si la plupart, lors de cet exercice oral, accentuent les différences entre les personnages déjà relevées dans l'écrit individuel de Valérie, l'interprétation par Émeline du personnage de Kamel ne laisse pas la classe indifférente. Elle campe un Kamel à la diction hachée, presque haletante. Youssef réagit aussitôt : «Kamel n'est peut-être 
pas aussi fort que ça!». Pour lui, le contenu des propos du jeune homme, en apparence très sûr de lui, masque une peur intérieure aussi intense que celle de Farid. Cette possible compréhension du personnage sera entérinée par une partie de la classe à la fin de ce débat interprétatif, y compris par Valérie. Il est d'ores-et-déjà intéressant pour nous d'observer que le passage par la mise en voix et en espace n'a pas donné lieu à une interprétation caricaturale des personnages, mais qu'elle semble avoir "rapproché » les élèves des personnages et complexifié leur approche de ceux-ci par une lecture plus fine du contenu de leurs répliques. Pour certains, "l'effetpersonnage ", même si cette expression est appliquée par V. Jouve (Ibid.) au roman, semble fonctionner à plein. Pour d'autres, qui le verbalisent moins, cela semble moins évident. Pour continuer l'exploration des effets de lecture permis par la construction des personnages, nous faisons alors le choix d'organiser, après la découverte de la suite de la pièce, une séance 4 permettant de confronter la situation de nos deux protagonistes à celles des migrants du roman de A. Ndione, Mbëkë mi. À l'assaut des vagues de l'Atlantique (2008 : 40-43), par le relevé des ressemblances et des différences entre les deux situations, opéré par les élèves. En effet, les deux extraits ont été choisis pour leur grande proximité thématique : ils mettent en scène des migrants africains s'apprêtant à embarquer de nuit. En leur proposant un extrait de roman verbalisant explicitement les sentiments des personnages, nous souhaitions lever les implicites du texte de M. Bey restés obscurs pour une partie des élèves. Dans l'extrait de roman, l'un des personnages exprime son inquiétude tandis que d'autres se moquent de lui pour mieux masquer leur propre peur. Tous les élèves sont ainsi amenés à mesurer les différences d'ordre générique entre les deux textes, et à approfondir leur compréhension du caractère des protagonistes théâtraux.

Cet approfondissement de l'approche psychologique des personnages devait servir une prise de recul quant aux nécessités dramaturgiques de la pièce. Au-delà de l'implication des élèves dans leur lecture, l'effet-personnage s'impose ici comme une nécessité dans la construction de la pièce. Le caractère antithétique des deux migrants assure la progression de l'intrigue. Les élèves se sont montrés parfois agacés par le personnage de Farid, moins sûr de lui, pourtant, ils ont réalisé, en découvrant le dénouement de la pièce et le péril qui guette les personnages, que le maintien de son inquiétude était nécessaire sur le plan dramaturgique.

\section{Favoriser la prise de recul des élèves sur la dimension idéologique de toute production littéraire}

Pourtant, à l'issue de la séance 4, faire réfléchir les élèves à la stratégie élaborée par M. Bey pour "mettre le lecteur dans son camp ", en lui permettant de se glisser dans la peau de ces jeunes gens et d'être touché par eux - sans qu'ils soient d'emblée classés au mieux dans la catégorie un peu abstraite des victimes, au pire dans celle d'étrangers entretenant peu de points 
communs avec le lecteur - nous parait à ce stade un peu trop complexe. Nous prenons le parti de passer, comme dit plus haut, par une nouvelle comparaison, avec un texte profondément ironique, "Baignade interdite " de P. Claudel (2015) pour mesurer l'écart de traitement subi par les migrants lors de leur arrivée sur les côtes européennes. Les élèves vont ainsi pouvoir confronter leur empathie pour les personnages de la pièce avec l'indifférence, voire le mépris, des personnages de P. Claudel qui se contentent de porter un regard extérieur et déshumanisant sur ces mêmes migrants. Le texte est assez complexe puisqu'il présente un dialogue entre un badaud habitué à venir observer, de loin, les bateaux de migrants depuis une plage, et un personnage qui le questionne, novice en la matière, mais enclin au même comportement. Les premières réactions fusent : "Ils n'ont jamais parlé à un migrant, ils viennent juste se divertir ", s'écrie Marie. Youcef renchérit : "Ils n'en ont vu qu'à la télé. Ils sont racistes, on dirait qu'ils se moquent ". Cette dernière observation rendant compte de la perception de l'ironie du texte n'est pas reprise par le reste des élèves. Nous décidons alors de différer ce questionnement pour laisser à chacun la possibilité d'expliciter ce qu'il a compris du texte sans valider d'emblée cette interprétation intéressante. La remarque de Youcef est cependant l'occasion d'éclaircir les définitions du racisme et de la xénophobie. Pour Aminata, les personnages « disent plein de clichés sur les migrants ». Quant à Lucie, elle se dit choquée par la phrase : "Ce qui est beau c'est de les voir apparaitre à l'horizon. Debout. Tous. Sur de maigres embarcations. Serrés les uns contre les autres. On dirait qu'ils marchent sur l'eau " (Claudel 2015: 84) : "Ils parlent d'eux comme si c'était un tableau, c'est sadique ", dit-elle. En présentant ce texte aux élèves, nous savions bien qu'ils ne manqueraient pas de réagir. Même si toutes les réactions n'étaient pas virulentes, tous les élèves condamnaient grosso modo les personnages, ou au moins l'un d'entre eux, celui du «badaud habitué ». Les phrases "Pourquoi venir ici ? Je veux dire spécialement ici ? Sans doute pensent-ils que c'est mieux que là-bas. Les pauvres. Comme vous dites " (Ibid. : 84) ont toutefois mené à un débat entre les élèves défendant une vision empathique des personnages (se raccrochant à l'expression « les pauvres ", en dépit d'un texte assez outrancier par ailleurs) et ceux plus sensibles à leur hypocrisie. Mais tout l'enjeu à présent était de les emmener sur le terrain des stratégies auctoriales. Pourquoi P. Claudel avait-il donc choisi de tels personnages ? Poser ainsi la question aurait d'emblée évincé une difficulté, celle d'une confusion possible entre le propos des personnages et celui de l'auteur. Lors de l'avant-dernière séance, nous choisissons plutôt de projeter au tableau une série d'assertions, déjà formulées en classe par certains d'entre eux. Nous modifions sciemment les deux dernières pour permettre aux élèves de prendre du recul et peut-être mieux cerner l'ironie du passage. À charge, donc à présent et pour tous les élèves, en groupes de trois ou quatre, de valider ou d'invalider ces affirmations en justifiant leurs réponses : 
Ils n'ont jamais parlé à un migrant. Ils viennent se divertir. C'est sadique l'histoire du tableau.

Ils disent plein de clichés sur les migrants, on croit qu'ils ont pitié mais ils font juste semblant, ils se moquent.

Les personnages et Philippe Claudel sont racistes.

L'auteur se moque des personnages qui observent les migrants.

Lors de la mise en commun, la discussion bat son plein. Aïcha précise ainsi : "Philippe Claudel va trop loin, il en rajoute, ça se fait pas, il était pas obligé d'aller jusque-là, je pense qu'il est raciste. " Alors que Youcef rétorque : «S'il était raciste, il aurait jamais écrit dans ce livre».

Le paratexte, judicieusement mobilisé par l'élève ici, avait été simplement indiqué sur le polycopié du texte sans que nous le commentions plus avant, mais le titre du recueil (Bienvenue! 34 auteurs pour les réfugiés) se présente effectivement en faveur des réfugiés.

Indira renchérit : " Il y a des auteurs qui écrivent sur des meurtriers, et ils sont pas forcément des meurtriers".

Moue sceptique d'Aïcha. C'est Émeline qui finira par la mettre sur la voie en la faisant changer d'avis : "C'est exagéré justement, du coup on peut pas croire à ce qu'ils disent ». Indira concluant : « Oui, ils sont trop débiles les personnages, c'est pas possible ".

La transition est alors toute trouvée pour envisager la dernière proposition, mais les avis sont là encore très tranchés.

Alexandre : "Non, l'auteur ne se moque pas d'eux »

M. : « Pourquoi dis-tu cela?»

Chimène : «Mais si ! Il se moque, parce qu'il leur fait dire des choses sur les migrants qui sont pas bien, ils racontent des choses sur eux qui sont pas vraies".

Lucie adopte, quant à elle, un moyen terme : «Il ne se moque pas d'eux mais il les dénonce pas non plus ".

Une mise au point sur la dénonciation implicite, par l'ironie notamment, et la dénonciation explicite (polémique, par exemple) est alors envisageable, préparant l'étude des registres qui sera systématisée au lycée. Les élèves relèvent ainsi particulièrement les expressions suivantes : "On dirait qu'ils marchent sur l'eau. Comme le Christ » (Ibid. : 84) ; "On ne parle pas leur langue et eux ne parlent pas la nôtre. Vous leur avez demandé ? Non mais ça se voit "; " De toute façon ils claquent tellement des dents qu'ils seraient incapables d'articuler un seul mot " (Ibid. : 84-85).

Le passage par le texte de P. Claudel permet en dernière séance de revenir sur les choix d'écriture de M. Bey, en comparant les stratégies des deux auteurs, concernant notamment le choix d'une écriture faisant la part belle à l'implicite. Les premières pistes d'interprétation de la situation des personnages, formulées en séance 1 par certains élèves, sont alors projetées à nouveau au tableau : 
Ils rentrent chez eux, ils ont la permission de minuit, mais ils ont peur de leur père.

Ils sont perdus.

Ils sont en danger.

Ils préparent un braquage.

Ils vont acheter de la drogue.

Ces affirmations sont assorties de la question suivante : "Pourquoi Maïssa Bey ne nous a-t-elle pas expliqué tout de suite la situation des personnages? ", les élèves devant formuler leurs réponses individuellement et par écrit.

Si quelques élèves, à l'instar de Cumba et Alexandre, produisent des réponses un peu attendues et générales telles que " éveiller notre curiosité " ou " pour laisser du suspense », la plupart parviennent à formuler une réponse méta-réflexive assez fine : " pour montrer qu'ils sont égaux et pareils à nous " (Shangari), " pour qu'au début on ait une autre image de Kamel et Farid et que ça nous choque quand on apprend que ce sont des migrants " (Youcef.), "pour éviter les préjugés" (Marie). Ils peuvent alors mieux appréhender la fin du texte qui voit les deux jeunes gens se mettre à courir vers le passeur et le bateau, et l'usage final de la voix-off signalant que la pièce s'inspire d'un fait divers survenu le 14 aout 2002 : "Ils étaient trois, trois jeunes gens d'environ 20 ans, à s'être embarqués clandestinement, dans la nuit du 14 au 15 aout, sur un bateau battant pavillon chinois, le " Jing Hong Hai » dans le port d'Oran. Découverts le lendemain par l'équipage, ils ont été ligotés et jetés en haute mer" (Bey: 43).

À la question suivante: "Pourquoi choisit-elle d'arrêter son texte au moment où ils courent vers le bateau ?", Iris et Marie répondent en se focalisant sur la révélation de la voix-off : «C'est plus brutal, on s'est attaché au personnage ", "ça rend la fin plus tragique ". Là où Yanis, qui comprend mieux la question parvient à écrire : "Pour qu'on se crée nous-mêmes la fin de l'histoire ", réponse qui entérine le caractère fictionnel des personnages et leur universalité aux yeux du lecteur.

La dernière étape de ce travail de prise de conscience de la dimension idéologique des textes consistait à remplir un bilan personnel :

Dans cette pièce de Maïssa Bey, le lecteur découvre une autre image des migrants $^{8}$. À travers les personnages de Farid et Kamel, le lecteur réalise que

Si l'auteur parvient à rendre cela possible, c'est grâce à

Les élèves ont su s'emparer de ce questionnement en mentionnant l'âge des personnages (" grâce à la jeunesse des personnages, on comprend mieux, on a plus pitié, on est plus sensibles aux jeunes qu'aux personnes âgées »écrit Yassine M.), ou encore leurs préoccupations adolescentes et universelles (les rêves, le football, les filles) : "les migrants sont des personnes comme nous.

8. Que celle que font apparaitre les personnages du texte de P. Claudel. 
Le texte nous montre leur vie, il nous fait les connaitre et nous montre qu'ils ne sont pas différents de nous et qu'ils ont même des points communs avec nous" (Edwine). Aïcha explique quant à elle que M. Bey "montre que les migrants ne sont pas comme on les voit à la télé, elle les présente comme des êtres humains qui ont seulement besoin d'aide et d'abri. Elle cherche à toucher les lecteurs, à leur donner une bonne image des migrants pour toucher notre sensibilité/solidarité ». Irina va dans le même sens. Pour elle, Maïssa Bey nous donne à lire " leurs comportements, leurs pensées. Ils ont des doutes, des envies aussi, et des raisons de partir et l'auteur réussit à nous identifier aux personnages et on voit à quel point ça doit être dur de quitter son pays ". Ces quelques exemples rendent compte de la tendance interprétative unanime de la classe en la matière et montrent une réflexion éthique et citoyenne, toujours appuyée sur l'analyse littéraire et métadiscursive des choix auctoriaux opérés.

\section{Au terme de la séquence...}

De la lecture stimulante de cette pièce et du cheminement intérieur qu'elle permet (autorisant, par son implicite, le lecteur à investir le texte des valeurs qui sont les siennes, de prime abord, afin de mieux les questionner par la suite), il nous semble qu'une éthique de la responsabilité a pu être construite sans instrumenter le texte. Il nous semble également, à l'échelle du groupe classe, que les stratégies auctoriales sont devenues une catégorie objectivable et questionnable par les élèves et que c'est en ce sens que ceux-ci peuvent apprendre à mieux analyser les discours explicitement idéologiques ou non. Il faudrait à présent étudier de près les discours fonctionnant au sein des représentations médiatiques afin d'en montrer la complexité et les nuances, car les médias ont souvent été mobilisés comme repoussoirs par les discours des élèves et le texte de $\mathrm{P}$. Claudel. Toutefois le trajet réalisé pendant cette séquence nous semble déjà avoir été conséquent.

\section{Virginie BRINKER \& Sandrine MESLET}

\section{Références bibliographiques}

- BEY, M. (2013). Tu vois c'que j'veux dire? Montpellier : Chèvre feuille étoilée.

- BISHOP, M.-F. \& ROUXEL, A. (dir.) (2007). « Sujet lecteur, sujet scripteur, quels enjeux pour la didactique? ". Le français aujourd'hui, 157.

- CLAUDEL, P. (2015). Baignade interdite. In Bienvenue! 34 auteurs pour les réfugiés (pp. 83-86). Paris : Points.

- GOIGOUX, R. \& CÈBE, S. (2009). Lector \& Lectrix. Apprendre à comprendre les textes narratifs. Paris : Retz.

- JOOLE, P. (2008). Comprendre des textes écrits, cycle 3/collège. Versailles : CRDP de l'académie de Versailles \& Retz.

- JOUVE, V. (1992). L'Effet-personnage dans le roman. Paris : Presses universitaires de France. 
- KANDÉ, S. (2011). La Quête infinie de l'autre rive. Paris: Gallimard, coll. "Continents noirs".

- LEGARDEZ, A. \& SIMONNEAUX, L. (dir.) (2006). L'École à l'épreuve de l'actualité. Enseigner les questions vives. Nogent-le-Rotrou : E.S.F.

- MAZAURIC, C. (2012). Mobilités d'Afrique en Europe. Récits et figures de l'aventure. Paris : Karthala.

- NDIONE, A. (2008). À l'Assaut des vagues de l'Atlantique. Paris : Gallimard, coll. "Continents noirs".

- ROUXEL, A. \& LANGLADE, G. (dir.) (2004). Le Sujet lecteur, lecture subjective et enseignement de la littérature. Rennes: Presses universitaires de Rennes.

- TAUVERON, C. (2004). La lecture, voie possible de (ré)conciliation des élèves en difficulté avec la lecture. In La Lecture et la culture littéraires au cycle des approfondissements, Actes de l'université d'automne, Clermont-Ferrand - Royat, du 23 au 31 octobre 2002, Versailles : SCÉRÉN - CRDP de l'académie de Versailles, coll. "Les Actes de la Desco ». 